\title{
Endophytic Microbiome Variation Among Single Plant Seeds
}

\author{
A. F. Bintarti, ${ }^{1}$ A. Sulesky-Grieb,, ${ }^{2,3}$ N. Stopnisek, ${ }^{1,2}$ and A. Shade ${ }^{1,2,3,4, \dagger}$ \\ ${ }^{1}$ Department of Plant, Soil and Microbial Sciences, Michigan State University, East Lansing, MI 48824 \\ ${ }^{2}$ Department of Microbiology and Molecular Genetics, Michigan State University, East Lansing, MI 48824 \\ ${ }^{3}$ Program in Ecology, Evolution and Behavior, Michigan State University, East Lansing, MI 48824 \\ ${ }^{4}$ The Plant Resilience Institute, Michigan State University, East Lansing, MI 48824
}

Accepted for publication 9 August 2021.

\begin{tabular}{|c|c|}
\hline \multicolumn{2}{|c|}{ ABSTRACT } \\
\hline $\begin{array}{l}\text { Like other plant compartments, the seed harbors a } \\
\text { microbiome. The members of the seed microbiome are the first } \\
\text { to colonize a germinating seedling, and they may initiate the } \\
\text { trajectory of microbiome assembly for the next plant generation. } \\
\text { Therefore, the members of the seed microbiome are important } \\
\text { for the dynamics of plant microbiome assembly and the vertical } \\
\text { transmission of potentially beneficial symbionts. However, it } \\
\text { remains challenging to assess the microbiome at the individual } \\
\text { seed level (and, therefore, for the future individual plants) due to } \\
\text { low endophytic microbial biomass, seed exudates that can } \\
\text { select for particular members, and high plant and plastid } \\
\text { contamination of resulting reads. Here, we report a protocol for } \\
\text { extracting microbial DNA from an individual seed (common } \\
\text { bean, Phaseolus vulgaris) with minimal disruption of host tissue, } \\
\text { which we expect to be generalizable to other medium- and } \\
\text { large-seed plant species. We applied this protocol to determine } \\
\text { the } 16 S \text { ribosomal RNA (rRNA) V4 and rRNA internal } \\
\text { transcribed spacer } 2 \text { amplicon composition and examine the }\end{array}$ & $\begin{array}{l}\text { variability of individual seed harvested from replicate common } \\
\text { bean plants grown under standard, controlled conditions to } \\
\text { maintain health. Using DNA extractions from individual seeds, } \\
\text { we compared seed-to-seed, pod-to-pod, and plant-to-plant } \\
\text { microbiomes, and found the highest microbiome variability at } \\
\text { the plant level. This suggests that several seeds from the same } \\
\text { plant could be pooled for microbiome assessment, given } \\
\text { experimental designs that apply treatments at the parent plant } \\
\text { level. This study adds protocols and insights to the growing } \\
\text { toolkit of approaches to understand the plant-microbiome } \\
\text { engagements that support the health of agricultural and } \\
\text { environmental ecosystems. }\end{array}$ \\
\hline
\end{tabular}

Seed microbiomes offer a reservoir of microbiota that can be vertically passed from parent plants to offspring (Mitter et al. 2017; Shade et al. 2017; Truyens et al. 2015), and some of these members have plant-beneficial phenotypes (Adam et al. 2018; Berg and Raaijmakers 2018; Bergna et al. 2018; López-López et al. 2010).

\section{Corresponding author: A. Shade; shadeash@msu.edu}

Funding: This research was supported by the United States Department of Agriculture (USDA) National Institute of Food and Agriculture (NIFA) grant 2019-6701929305. A. Shade acknowledges support from the USDA-NIFA and Michigan State University AgBioResearch (Hatch). A. F. Bintarti acknowledges a doctoral fellowship from the Fulbright Foundation and from Michigan State University. N. Stopnisek acknowledges support from the Michigan State University Plant Resilience Institute.

*The $\boldsymbol{e}$-Xtra logo stands for "electronic extra" and indicates that a supplementary table and supplementary materials are published online.

The author(s) declare no conflict of interest.

(C) 2022 The American Phytopathological Society
Therefore, the seed microbiome is expected to play a key role in plant health and fitness (Barret et al. 2015), and especially in the assembly and establishment of the developing plant's microbiome (Chesneau et al. 2020). This expected importance of the seed microbiome has fueled recent research activity to use high-throughput sequencing to characterize the seed microbiomes of various plants (Chartrel et al. 2021; Dai et al. 2020; Eyre et al. 2019; Raj et al. 2019; Rodríguez et al. 2020; Xing et al. 2018).

Seed microbiomes include microbial members that live on the seed surface as epiphytes and members that colonize inside the internal tissues of the seed as endophytes (Nelson 2018). Among these microbiome members, endophytes that closely associate with endosperm and embryo tissues are more likely to be transmitted to the next plant generations than are seed-associated epiphytes (Barret et al. 2016; Nelson 2018). By itself, an endophytic association does not confirm that there is a functional benefit or coevolutionary relationship between the plant and the microbiome member (Nelson 2018). However, endophytic microbes offer the first source of inoculum for the germinating seedling (Nelson 2018; Vujanovic and Germida 
2017) and, given the potential for priority effects or pathogen exclusion, these members can have implications for the mature plant's microbial community composition or structure. Therefore, understanding the endophytic seed microbiome is expected to provide insights into how seeds can facilitate microbiome assembly and the vertical transmission of microbiome members over plant generations.

As is true for other plant compartments, different plant species or divergent crop lines, varieties, or cultivars often have different seed microbiome composition (taxonomic identities of members) or structure (relative contributions of taxa to the community) (Johnston-Monje and Raizada 2011; Klaedtke et al. 2016; LópezLópez et al. 2010; Wassermann et al. 2019). However, many seed microbiome studies have reported generally high variability across seed samples from the same plant type and treatment (Bergna et al. 2018; Bintarti et al. 2020; López-López et al. 2010), with strong explanatory value of either seed origin or seed lot, geographic region, or soil edaphic conditions (Chartrel et al. 2021; JohnstonMonje and Raizada 2011; Klaedtke et al. 2016). Although these insights may call into question the proportion of "inherited" versus acquired seed microbiome members, the high microbiome variability may be due, in part, to methods applied to extract the microbial DNA from the seed compartment, and different methods applied across studies. For instance, some studies surface sterilize the seed while others do not, some germinate the seed prior to microbiome analysis while others do not, and so on. One source of microbiome variability could be the common practice of the pooling of many seeds from the same or different plants to produce a composite seed microbiome sample for DNA extraction. Because multiple seeds are investigated at once, it is unclear at what level the most microbiome variability is highest: the seed, the pod or fruit, the plant, or the field or treatment. This information is required to determine the necessary sample size in well-powered experimental designs. More importantly, the question of vertical transmission cannot directly be addressed without seed microbiome assessment of an individual.

Our study objectives were to (i) determine the appropriate observational unit of endophytic seed microbiome assessment for common bean (Phaseolus vulgaris L.) by examining seed-to-seed, pod-to-pod, and plant-to-plant variability in $16 \mathrm{~S}$ ribosomal RNA (rRNA) V4 and rRNA internal transcribed spacer (ITS)2 amplicon analyses and (ii) develop a robust protocol for individual seed microbiome extraction that could be generally applied to other plants that have similarly medium- to large-sized seed. Here, we use a working definition of seed endophyte as the microbes internal to the ungerminated seed, including under the seed coat and within the internal compartments (cotyledon, radical, hypocotyl, and plumule) but excluding those on the surface of the seed coat. Our rationale for applying this working definition is to distinguish microbes that are more likely acquired via the parent plant from those that may have been acquired via the seed surface contact with the environment. We found that plant-to-plant variability under controlled growth conditions exceeded within-plant variability among different pods and conclude that seeds can be pooled by parent plant (but not across different plants) in study designs that aim to compare seed microbiomes resulting from treatments applied at the level of the individual plant (e.g., the experimental unit is one plant).

\section{MATERIALS AND METHODS}

Growth conditions for parent plants. We used common bean $P$. vulgaris L. 'Red Hawk', a dark red kidney bean developed at Michigan State University (Kelly et al. 1998) which belongs to the Andean lineage (Castro-Guerrero et al. 2016). The seed used to grow the parental plants originated from the Michigan State University's Agronomy Farm located in East Lansing, MI, U.S.A., and were harvested following standard agricultural practices.

Because we targeted the endophytic seed microbiome, surface sterilization of the bean seed was conducted before germination and planting. To sterilize, seed were soaked in a solution of $10 \%$ bleach with $0.1 \%$ Tween 20 for $15 \mathrm{~min}$, then rinsed four times with sterile water. The final rinse water was plated on tryptic soy agar and potato dextrose agar plates to test for sterilization efficacy. Sterilized seeds were placed in Petri dishes on sterile tissue paper moistened with sterile water, and allowed to germinate in the dark for 4 days. After 4 days, the radicle had emerged and the germinated seeds were transferred to the growth chamber. The germinated seeds were planted in three 4.54-liter (1-gal.) pots filled with a $50: 50(\mathrm{vol} / \mathrm{vol})$ mixture of agricultural bean field soil and vermiculite. The pots were placed in a BioChambers model SPC-37 growth chamber with a cycle of $14 \mathrm{~h} /$ day and $10 \mathrm{~h} / \mathrm{night}$ at 26 and $22^{\circ} \mathrm{C}$, respectively, $260 \mathrm{mE}$ light intensity, and $50 \%$ relative humidity. All plants received $300 \mathrm{ml}$ of water every other day and $200 \mathrm{ml}$ of half-strength Hoagland solution (Hoagland and Arnon 1950) once a week.

Study design. We planted three germinated seeds per pot and culled to one seedling per pot at the early vegetative growth stage. There were three plant replicates designated as A, B, and C, grown under the above-described conditions for normal, healthy growth. The three plants yielded different numbers of pods and seeds, and we aimed to balance and maximize the number of seeds used for analysis across plants (Table 1).

Seed harvest and endophyte microbial DNA extraction. Once the plants reached maturity at the R9 growth stage (yellowing leaves and dry pods), the seeds were harvested for endophytic microbiome analysis. Seeds were distinguished by plant and pod. The endophytic microbiome of each seed was extracted and sequenced individually. To extract the endophytic microbial DNA, a protocol was adapted from Barret et al. (2015) and Rezki et al. (2018). First, the seeds were surface sterilized as above and the

TABLE 1

Parent plant yield information and seed samples used in microbiome analyses

\begin{tabular}{|c|c|c|c|c|}
\hline \multirow[b]{2}{*}{ Plant } & \multicolumn{2}{|c|}{ Number produced ${ }^{\mathrm{a}}$} & \multicolumn{2}{|c|}{ Sequencing samples } \\
\hline & Pods & Seeds & Pods & Seeds \\
\hline \multirow[t]{3}{*}{ A } & 5 & 22 & A1 & $4^{c}$ \\
\hline & - & - & $\mathrm{A} 2$ & 4 \\
\hline & - & - & A3 & 4 \\
\hline \multirow[t]{6}{*}{ B } & 6 & 29 & B1 & $4^{c}$ \\
\hline & - & - & B2 & 4 \\
\hline & - & - & B3 & 4 \\
\hline & - & - & B4 & 4 \\
\hline & - & - & B5 & 4 \\
\hline & - & - & B6 & 4 \\
\hline \multirow[t]{3}{*}{ C } & 7 & 26 & C5 & 3 \\
\hline & - & - & C6 & 4 \\
\hline & - & - & $\mathrm{C} 7$ & 4 \\
\hline \multicolumn{5}{|c|}{$\begin{array}{l}\text { a Numbers of seeds per pod varied from } 2 \text { to } 6 \text {. } \\
\text { b Sequencing samples for } 16 S \text { ribosomal rRNA (rRNA) V4 and } \\
\text { rRNA internal transcribed spacer (ITS) } 2 \text { ( } n=47 \text { for bacteria or } \\
\text { archaea and } n=45 \text { for fungi) were grouped by plant. } \\
\text { c Unable to amplify rRNA ITS2 target DNA in one (of the four total) } \\
\text { seed samples. }\end{array}$} \\
\hline
\end{tabular}


seed coat was carefully removed using sterilized forceps. Each seed was then soaked in $3 \mathrm{ml}$ of phosphate-buffered saline solution with $0.05 \%$ Tween 20 (hereafter, "soaking solution") overnight at $4{ }^{\circ} \mathrm{C}$ with constant agitation of $170 \mathrm{rpm}$. Because low levels of microbial biomass are expected in single-seed extractions, positive and negative controls were included in the extraction protocol. This ensures that, if no extractable microbial DNA is present in a sample, it is representative of the sample rather than the extraction methods. A mock community was used as a DNA extraction positive control by adding one 75- $\mu \mathrm{l}$ aliquot of the ZymoBIOMICS Microbial Community Standard (Zymo Research, Irvine, CA, U.S.A.) to $3 \mathrm{ml}$ of the soaking solution immediately prior to conducting the extraction protocol. Sterile soaking solution $(3 \mathrm{ml})$ was used as a negative DNA extraction control.

After soaking overnight, the samples were centrifuged at $4,500 \times g$ for $60 \mathrm{~min}$ at $4^{\circ} \mathrm{C}$ to pellet any material that had been released from the seed tissues. After centrifugation, the seed was removed, and the pelleted material was resuspended in 1 to $2 \mathrm{ml}$ of supernatant (soaking solution) and transferred to a microcentrifuge tube for DNA extraction using the E.Z.N.A Bacterial DNA Kit (Omega Bio-tek, Inc. Norcross, GA, U.S.A.). The manufacturer's centrifugation protocol was used, with minor modifications. Specifically, the pelleted seed material was suspended in Tris-EDTA buffer (step 4), the incubation for the lysozyme step was extended to $20 \mathrm{~min}, 30 \mu \mathrm{l}$ of elution buffer was used, and the elution step was extended to a 15-min incubation. These modifications were performed to maximally recover the limited amount of microbial DNA expected from a single seed. We detail the standard operating protocol and provide notes on the alternatives that we tested in optimizing this protocol in the Supplementary Material.

PCR amplification and amplicon sequencing. To confirm successful DNA extraction from the seed pellet, DNA quantification and target gene PCR assays were performed. First, the DNA extracted from the seed samples and the positive and negative controls were quantified using the Qubit dsDNA BR Assay Kit (Thermo Fisher Scientific, Waltham, MA, U.S.A.). Then, PCR amplification and sequencing of the V4 region of 16S rRNA bacterial or archaeal gene and the ITS2 region of the fungal rRNA gene were performed. The V4 region of $16 \mathrm{~S}$ rRNA gene amplification was conducted using 515f (5'-GTGCCAGCMGCCGCGGTAA-3') and $806 \mathrm{r}$ (5'-GGACTACHVGGGTWTCTAAT-3') universal primers (Caporaso et al. 2011) under the following conditions: $94^{\circ} \mathrm{C}$ for $3 \mathrm{~min}$; followed by 35 cycles of $94^{\circ} \mathrm{C}$ for $45 \mathrm{~s}, 50^{\circ} \mathrm{C}$ for $60 \mathrm{~s}$, and $72^{\circ} \mathrm{C}$ for $90 \mathrm{~s}$; with a final extension at $72^{\circ} \mathrm{C}$ for $10 \mathrm{~min}$. The amplification was performed in $25-\mu \mathrm{l}$ mixtures containing $12.5 \mu \mathrm{l}$ of GoTaq Green Master Mix (Promega Corp., Madison, WI, U.S.A.), $0.625 \mu \mathrm{l}$ of each primer $(20 \mu \mathrm{M}), 2 \mu \mathrm{l}$ of DNA template (approximately $1 \mathrm{ng} / \mu \mathrm{l}$ ), and $9.25 \mu \mathrm{l}$ of nuclease-free water. The amplicon DNA (concentration of approximately $1 \mathrm{ng} / \mu \mathrm{l}$ ) was sequenced at the Research Technology Support Facility (RTSF) Genomics Core, Michigan State sequencing facility using the Illumina MiSeq platform v2 Standard flow cell. The sequencing was performed in a 2-by-250-bp paired-end format.

The PCR amplification of the rRNA ITS2 region was performed using ITS86f (5'-GTGAATCATCGAATCTTTGAA-3') and ITS4 $\left(5^{\prime}\right.$-TCCTCCGCTTATTGATATGC-3') primers (Op De Beeck et al. 2014), with the addition of index adapters by the RTSF Genomics Core. The PCR amplification of the rRNA ITS2 was conducted under the following conditions: $95^{\circ} \mathrm{C}$ for $2 \mathrm{~min}$; followed by 40 cycles of $95^{\circ} \mathrm{C}$ for $30 \mathrm{~s}, 55^{\circ} \mathrm{C}$ for $30 \mathrm{~s}$, and $72^{\circ} \mathrm{C}$ for $1 \mathrm{~min}$; with a final extension at $72^{\circ} \mathrm{C}$ for $10 \mathrm{~min}$. The amplification was performed in a 50- $\mu \mathrm{l}$ mixture containing $20 \mu \mathrm{l}$ of GoTaq Green Master Mix (Promega Corp.), $1 \mu \mathrm{l}$ of each primer $(10 \mu \mathrm{M}), 1 \mu \mathrm{l}$ of DNA template (approximately $1 \mathrm{ng} / \mu \mathrm{l}$ ), and $27 \mu \mathrm{l}$ of nuclease-free water. The PCR products were purified using a QIAquick PCR Purification Kit (Qiagen, Hilden, Germany). Purified PCR products with a concentration range of 6 to $10 \mathrm{ng} / \mu \mathrm{l}$ were sequenced at the RTSF Genomics Core using Illumina MiSeq platform v2 Standard flow cell and 2-by-250-bp paired-end format.

Sequence analysis. The USEARCH pipeline (v.10.0.240) was used to merge paired-end bacterial or archaeal raw reads, filter for low-quality sequences, dereplicate, remove singletons, denoise, and check for chimeras (Edgar and Flyvbjerg 2015). An in-house open reference strategy was performed for operational taxonomic unit (OTU) clustering (Rideout et al. 2014). First, closed-reference OTU picking was performed by clustering the quality filtered reads against the SILVA database (v.132) (Quast et al. 2013) at 97\% identity using the USEARCH algorithm (usearch_global command) (Edgar 2010). Then, a de novo OTU picking process was performed on the reads that failed to match the reference using UPARSE-OTU algorithm (cluster_otus command) (Edgar 2013) at 97\% identity. Finally, closed-reference and de novo OTUs were combined into a full set of representative sequences. The merged sequences were then mapped back to the representative sequences using the usearch_global command.

Sequence alignment, taxonomy assignment, nonbacterial or archaea filtering, and phylogenetic diversity calculation were performed using QIIME 1.9.1 (Caporaso et al. 2010b). The representative sequences were aligned against the SILVA database (v.132) (Quast et al. 2013) using PyNAST (Caporaso et al. 2010a). The unaligned OTUs and sequences were excluded from the OTU table and the representative sequences file, respectively. Taxonomy assignment was performed using the default classifier method (UCLUST algorithm) at a minimum confidence of 0.9 (Edgar 2010) using SILVA database (v.132) as the reference. Plant contaminants (chloroplast and mitochondria) and unassigned taxa were removed from the OTU table and the representative sequences using filter_ taxa_from_otu_table.py and filter_fasta.py command (Supplementary Fig. S1). Filtering the microbial contaminants from the OTU table was conducted in R (v.3.4.2; R Core Development Team) using the microDecon package (McKnight et al. 2019). Reads were normalized using the cumulative sum scaling (CSS) method in metagenomeSeq Bioconductor package in R (Paulson et al. 2013).

The fungal ITS raw reads were processed using the USEARCH (v.10.0.240) pipeline. Read processing included merging paired-end reads, removing primers using cutadapt (v.2.1) (Martin 2011), dereplication, and singleton removal. OTUs were picked and chimeras removed using de novo clustering at $97 \%$ identity threshold with the UPARSE-OTU algorithm (cluster_otus command) (Edgar 2013). Then, all merged sequences were mapped to the clustered reads using the usearch_global command to generate an OTU table. Fungal taxonomic classification was performed in CONSTAX (Gdanetz et al. 2017) using RDP Classifier (v.11.5) (Cole et al. 2014; Wang et al. 2007) at a minimum confidence of 0.6 and with the UNITE reference database (release 12 January 2017). Plant and microbial contaminants removal and read normalization were performed in $\mathrm{R}$ (v.3.4.2). Plant contaminants were removed from the OTU table by filtering out OTUs that were assigned into kingdom Plantae (Supplementary Fig. S1). Microbial contaminants were removed using the microDecon package (McKnight et al. 2019). The CSS method from the metagenomeSeq Bioconductor package was performed to normalize the fungal reads (Paulson et al. 2013).

Microbial community analysis. Microbiome statistical analyses were conducted in R (v.3.4.2) ( $\mathrm{R}$ Core Development Team). Microbial $\alpha$ and $\beta$ diversity were calculated on the CSS-normalized OTU table using the vegan package (v.2.5-7) (Oksanen et al. 2019). Richness (count of observed OTUs) and Faith's phylogenetic diversity were used to analyze the bacterial or archaeal $\alpha$ diversity. 
For fungal $\alpha$ diversity, we used richness. The evenness of the seed microbiomes was visualized using rank-abundance curves (Phyloseq package v.1.28.0) in R (McMurdie and Holmes 2013). Differences in $\alpha$ diversity among plants and pods were determined by fitting the linear mixed-effects model (LMM) using the lme function of the nlme package (version 3.1-152) (Pinheiro et al. 2021). We performed LMM because the study has an unbalanced nested design, with pod as the random factor nested within plant as the fixed factor. Microbial composition and relative abundance were analyzed using the Phyloseq package (v.1.28.0) in $\mathrm{R}$ (McMurdie and Holmes 2013).

$\beta$ Diversity was calculated using Jaccard distances and visualized using a principal coordinate analysis (PCoA) plot. We used the Jaccard index, which is based on presence or absence (unweighted), rather than a metric based on relativized abundance (weighted) because we reasoned that the seed microbiome members are likely to be dormant inside the seed prior to germination (Cope-Selby et al. 2017), and that any differences in relative abundances are not directly attributable to competitive fitness outcomes inside the seed. Furthermore, exponential growth would allow that any viable cell successfully packaged and passaged via the seed could, in theory, successfully colonize the new plant. Finally, consistent host selection or enrichment (that may favor some taxa over others) cannot be assessed directly with our experimental design because we do not have data from multiple plant generations. For comparison, we also provide an assessment of $\beta$ diversity using the weighted BrayCurtis dissimilarity (Supplementary Fig. S2) but caution against overinterpreting abundance-weighted analyses for the reasons listed above.

Nested permutational multivariate analysis of variance (PERMANOVA) using the function nested.npmanova from the BiodiversityR package (Kindt 2020) was performed to assess the microbial community composition and structure among plants and pods. We performed multivariate analysis to check the homogeneity of dispersion (variance) among groups using the function betadisper (Oksanen et al. 2019). We performed PERMDISP to test the significant differences in dispersions between groups and Tukey's honestly significant difference (HSD) test to determine which groups differ in relation to the dispersions (variances).

Power analysis and sample size were calculated using the pwr.t.test function from the pwr package (v.1.3-0). We performed power analysis of a two-category $t$ test. Because the most microbiome variability was observed across plants, we pooled individual seed sequence profiles in silico at the parent plant level for this analysis. We calculated Cohen's $d$ effect size given the information of mean (M) and standard deviation (SD) of bacterial or archaeal $\alpha$ diversity (richness and phylogenetic diversity) from three plant samples from this study: plant A $(n=12$; richness: $\mathrm{M}=30.58$, $\mathrm{SD}=6.42$, phylogenetic diversity: $\mathrm{M}=4.17, \mathrm{SD}=0.89$ ), plant $\mathrm{B}$ ( $n=24$; richness: $\mathrm{M}=18.21, \mathrm{SD}=7.35$, phylogenetic diversity: $\mathrm{M}=2.92, \mathrm{SD}=0.82)$, and plant $\mathrm{C}(n=11$; richness: $\mathrm{M}=19.09$, $\mathrm{SD}=10.95$, phylogenetic diversity: $\mathrm{M}=3.09, \mathrm{SD}=1.39$ ). We calculated the common SD ( $\sigma$ pool of all groups) using the above information; then, we calculated Cohen's $d$ effect size for both richness and phylogenetic diversity. Cohen's $d$ effect size was defined by calculating the difference between the largest and smallest means divided by the square root of the mean square error (or the common SD). Power analysis was run with Hedges's $g$ effect size (corrected with Cohen's $d$ effect size) and significance level of 0.05 .

Here, we defined shared microbiome members (sometimes referred to as a "core") as microbial taxa that were shared and detected in all considered samples. Taxon occupancy is the proportion of samples in which the taxa are detected, with an occupancy of 1 , meaning that the taxon was detected in all samples (Shade and Stopnisek 2019). We report the taxa that were shared across seeds originating from different plants and across seeds that originated from the same plant.

Data and code availability. The computational workflows for sequence processing and ecological statistics are available on GitHub (https://github.com/ShadeLab/Bean_seed_variability_Bintarti_ 2021). Raw sequence data of bacteria or archaea and fungi have been deposited in the Sequence Read Archive NCBI database under Bioproject accession number PRJNA714251.

\section{RESULTS}

Sequencing summary and microbiome coverage. In total, $5,056,76916 \mathrm{~S}$ rRNA V4 and 8,756,009 rRNA ITS2 quality reads were generated from 47 DNA samples isolated from individual seeds for bacteria or archaea, and from 45 samples for fungi. We removed $>90 \%$ of reads that were plant contaminants (Supplementary Fig. S1), resulting in 17,128 and 67,878 16S rRNA bacterial or archaeal and rRNA ITS2 fungal reads, respectively. After removing plant and microbial contaminants, we determined 211 bacterial or archaeal and 43 fungal OTUs defined at $97 \%$ sequence identity. Although the majority of individual seeds from plants A and B had exhaustive to sufficient sequencing effort, some seeds from plant $\mathrm{C}$ did not (Fig. 1A). However, the fungal rarefaction curves reached asymptotes and had sufficient sequencing depth (Fig. 1B). Both bacterial or archaeal and fungal seed microbiomes were highly uneven, with few dominant and many rare taxa, as is typical for microbiomes (Fig. 1C and D).

Microbiome diversity. There were differences in bacterial or archaeal community richness among seeds from different plants $(\mathrm{LMM} ; \mathrm{df}=2, F$ value $=6.91, P$ value $=0.015)($ Fig. $2 \mathrm{~A})$, where plant B and C had lower seed richness than plant A (Tukey's HSD post hoc test; $P$ value $=0.001$ and 0.006 , respectively). However, bacterial or archaeal community richness among seeds from pods collected from the same plant were not different (LMM, $P$ value $>$ 0.05 ) (Fig. 2B). Similarly, bacterial or archaeal phylogenetic diversity were different among seeds collected from different plants $($ LMMs; $\mathrm{df}=2, F$ value $=6.56, P$ value $=0.003)($ Fig. $2 \mathrm{C})$ but not among seeds from pods within the same plant (LMM, $P$ value $>$ 0.05) (Fig. 2D). Plants B and C had lower seed microbiome bacterial or archaeal phylogenetic diversity compared with plant A (Tukey's HSD post hoc test, $P$ value $=0.001$ and 0.013 , respectively). We observed no differences in fungal richness among seeds from different plants $(\mathrm{LMM}$; df $=2, F$ value $=1.11, P$ value $=$ 0.37 ) (Fig. 2E) and among seeds from pods within the same plant (LMM, $P$ value $>0.05$ ) (Fig. 2 F). To summarize, these results suggest that seed bacterial or archaeal $\alpha$ diversity but not fungal diversity varied plant to plant.

We detected a difference in seed bacterial or archaeal community composition among plants (nested PERMANOVA, df $=2$, $F$ value $=2.94, R^{2}=0.12, P$ value $\left.=0.002\right)($ Fig. $3 \mathrm{~A})$ but, again, not among pods from the same plant (nested PERMANOVA, $\mathrm{df}=$ $9, F$ value $=0.99, R^{2}=0.18, P$ value $=0.63$ ). Though separation among pods and plants are not obvious on the PCoA for the fungal seed microbiomes, we detected modest differences in fungal community composition among seeds from different plants (nested PERMANOVA, $\mathrm{df}=2, F$ value $=1.69, R^{2}=0.09, P$ value $\left.=0.004\right)$ (Fig. 3B), but not among seeds from pods from the same plant (nested PERMANOVA, df $=9, F$ value $=1.17, R^{2}=0.25, P$ value $=0.11$ ) An analysis of $\beta$ dispersion revealed that there were differences in seed microbiome dispersion across different plants for bacterial or archaeal communities (PERMDISP, $\mathrm{df}=2, F$ value $=63.94, R^{2}=$ $0.74, P$ value $=0.001$ ) (Fig. $3 \mathrm{C}$ ) but not for fungal communities $\left(\right.$ PERMDISP, df $=2, F$ value $=0.89, R^{2}=0.05, P$ value $\left.=0.4\right)$ 
(Fig. 3D). These results are qualitatively the same for analyses based on community structure as assessed by Bray-Curtis dissimilarity (Supplementary Fig. S2). Therefore, statistical differences in the seed microbiome across plants for the bacteria or archaea may be attributed to either centroid or dispersion, whereas fungal seed communities were different by centroid.

Bean seed microbiome composition. We identified 135 bacterial or archaeal and 49 fungal taxa at the genus level. The bacterial or archaeal individual seed communities were dominated by taxa from classes Gammaproteobacteria (50.47\%), Bacilli (24.48\%), Alphaproteobacteria (8.68\%), and Bacteroidia (6.59\%) (Fig. 4A), and include Pseudomonas (13.58\%), Bacillus (10.2\%), Acinetobacter (9.5\%), Raoultella (7.09\%), and Escherichia-Shigella $(5.19 \%)$ as the major genera. Among members of the class Alphaproteobacteria, we also found genera Bradyrhizobium and Allorhizobium-Neorhizobium-Pararhizobium-Rhizobium, with relative abundance of 2.57 and $0.85 \%$, respectively. Although seed fungal community composition varied among plants and also pods within plant, the fungal community was dominated by taxa belonging to classes Dothideomycetes (22.77\%), Agaricomycetes (16.61\%), and Eurotiomycetes (14.44\%) and the genera Aspergillus
(14.44\%), Capnodiales unidentified sp. 23791 (9.27\%), and Aureobasidium $(8.28 \%)$.

A key objective of this research was to understand the sources of variability in the individual bean seed microbiome to inform future study design. Because we found that the plant-to-plant seed microbiome variability was highest when grown in control conditions, we performed a power analysis to determine how many plants would be required to observe a treatment effect from seed samples pooled per plant. To detect the effect of treatment on bacterial or archaeal richness and phylogenetic diversity, pooled seeds from 9 and 12 plants are needed for $16 \mathrm{~S}$ rRNA richness and phylogenetic diversity, respectively, to achieve power of 0.8 ; and 13 and 19 plants to achieve power of 0.95 (Fig. 5).

Shared taxa among seeds and plants. We explored the microbial taxa shared across all seed samples, detected across all three plants, and also shared among all seeds within one plant (Supplementary Table S1). Although there were no bacterial or archaeal taxa detected and shared among all seeds, there were 11 taxa detected in more than half of seed samples (occupancy $>0.5, n=$ 47), and taxa from genus Bacillus were most common. Other bacterial or archaeal taxa found in more than half of seeds were

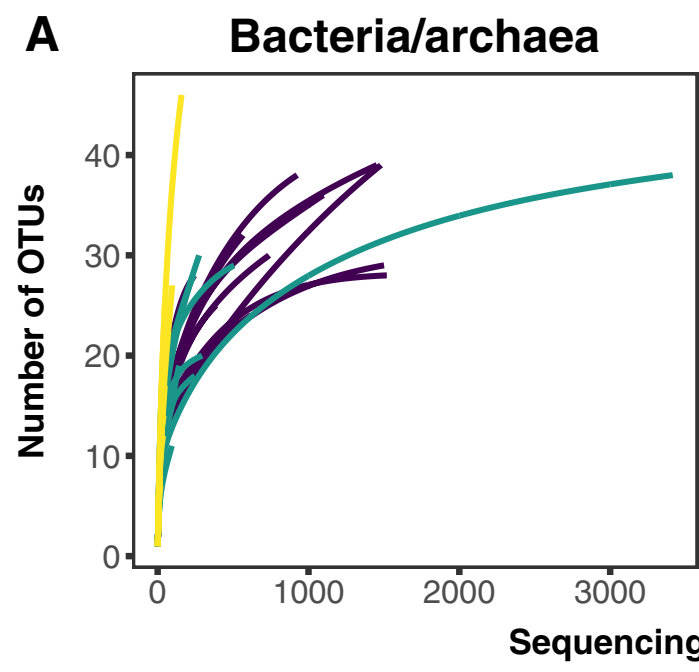

B

Fungi

C
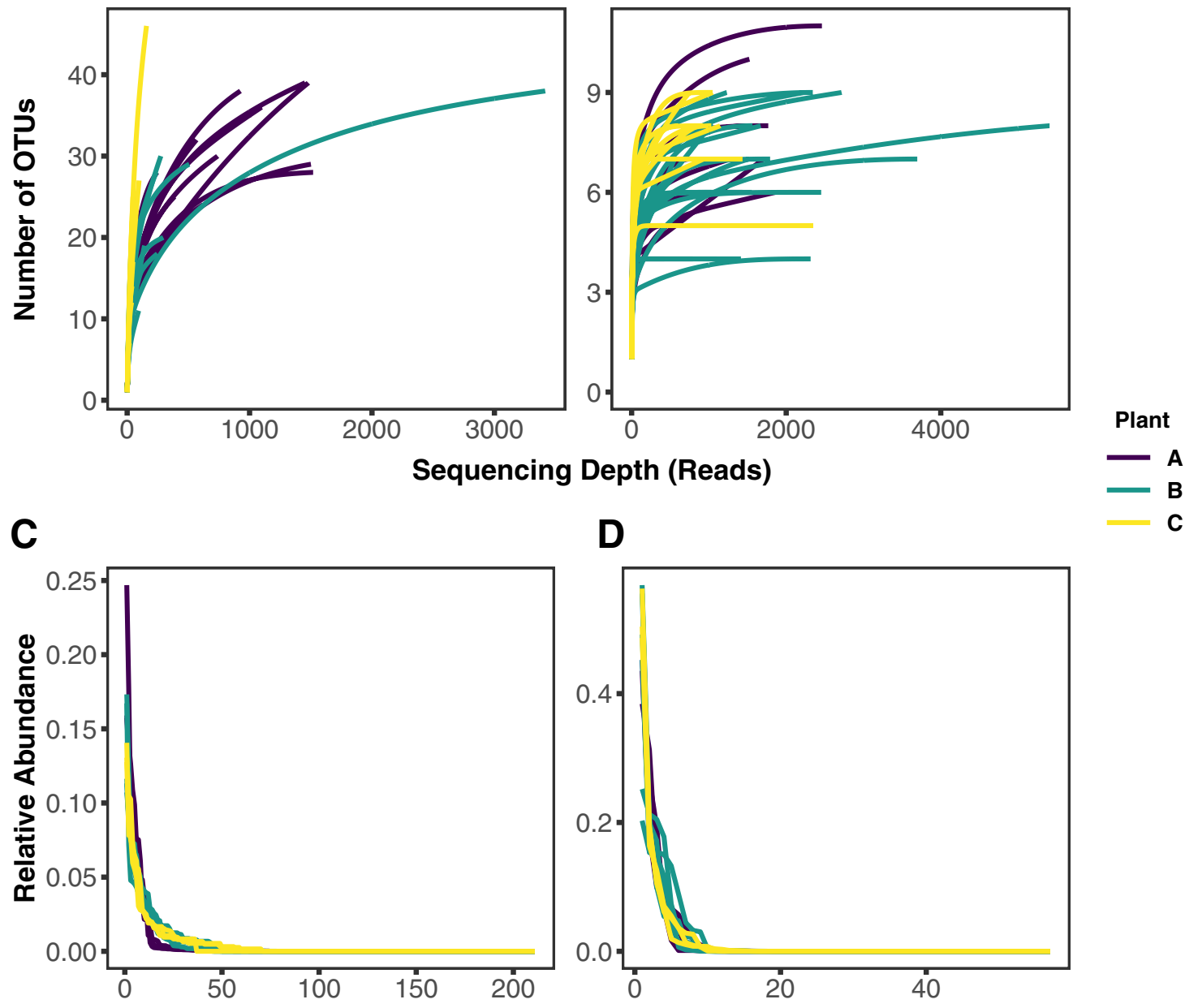

D

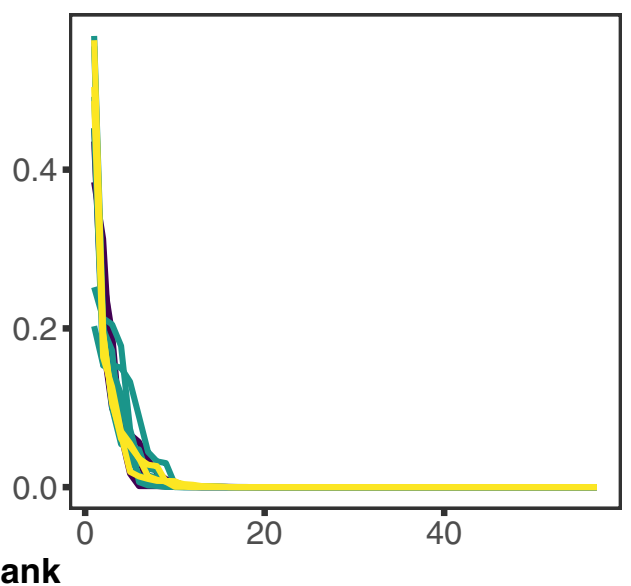

Fig. 1. Rarefaction curves of A, bacteria or archaea and B, fungi from individual seeds (marked) at $97 \%$ of clustering threshold were constructed by plotting the operational taxonomic unit (OTU) number after decontamination (microbial and plant contaminants removed) to the sequence (read) number. Each curve represents microbiome sequence data from microbial DNA extraction from an individual seed. Rarefaction curves were constructed using the vegan package (v2.5-4). Rank abundance curve of decontaminated and normalized C, bacterial or archaeal and D, fungal OTU tables. Samples ( $n=47$ and $n=45$ for bacteria or archaea and fungi, respectively) were grouped by plant. 
assigned to the genera Stenotrophomonas, Raoultella, Pseudomonas, Lactobacillus, Acinetobacter, Listeria, Bradyrhizobium, and Enterococcus. There were no fungal taxa shared among seeds. One fungal taxon from the genus Aspergillus was detected in $\sim 30 \%$ of the seeds.

In all, 54 bacterial or archaeal taxa were detected and shared across all plants, and these belonged to phyla Proteobacteria (Gammaproteobacteria $=21$ OTUs, and Alphaproteobacteria $=$ 6 OTUs), Firmicutes (13 OTUs), Actinobacteria (7 OTUs), Acidobacteria (4 OTUs), Chloroflexi (1 OTU), Bacteroidetes (1 OTU), and Verrucomicrobia (1 OTU). There were seven fungal taxa detected and shared across all plants, and these belonged to classes Eurotiomycetes (1 OTU), Dothideomycetes (2 OTUs), Sordariomycetes (1 OTU), Malasseziomycetes (1 OTU), Agaricomycetes (1 OTU), and one unindentified fungal taxon (Supplementary Table S1). Together, these results suggest the taxa that should be explored further to understand any importance to the host and their consistency and rates of transmission from plant parent to offspring.

\section{DISCUSSION}

There remain gaps in our understanding of the persistence and assembly of seed microbiome members, especially across plant generations, and which microbiome members are beneficial and actively selected by, or even coevolved with, the host. Here, we investigated the variability of the common bean microbiome at the resolution of the individual seed, which is the unit that delivers any vertically transmitted microbiome to the offspring. Because multiple legume seeds within a pod develop as a result of a single flower pollination, one simple hypothesis is that the individual seeds within a pod may harbor a highly similar microbiome if the floral pathway of assembly

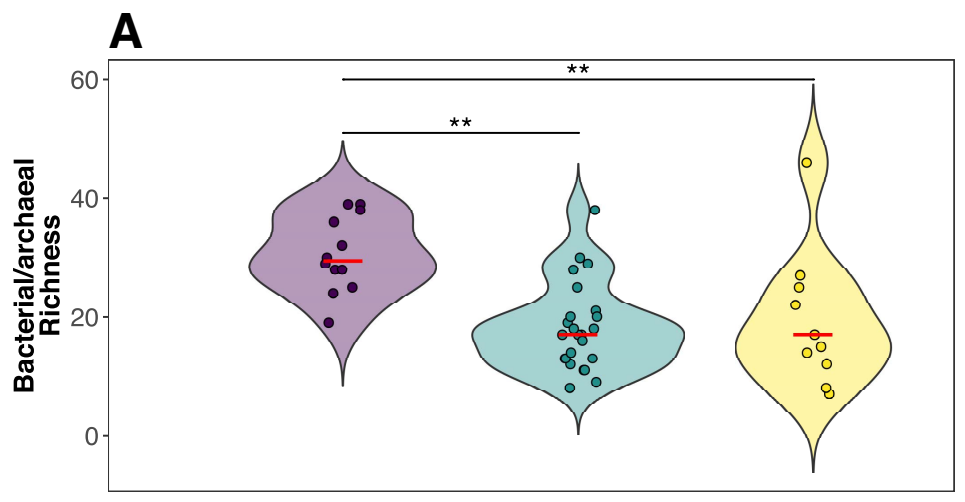

B
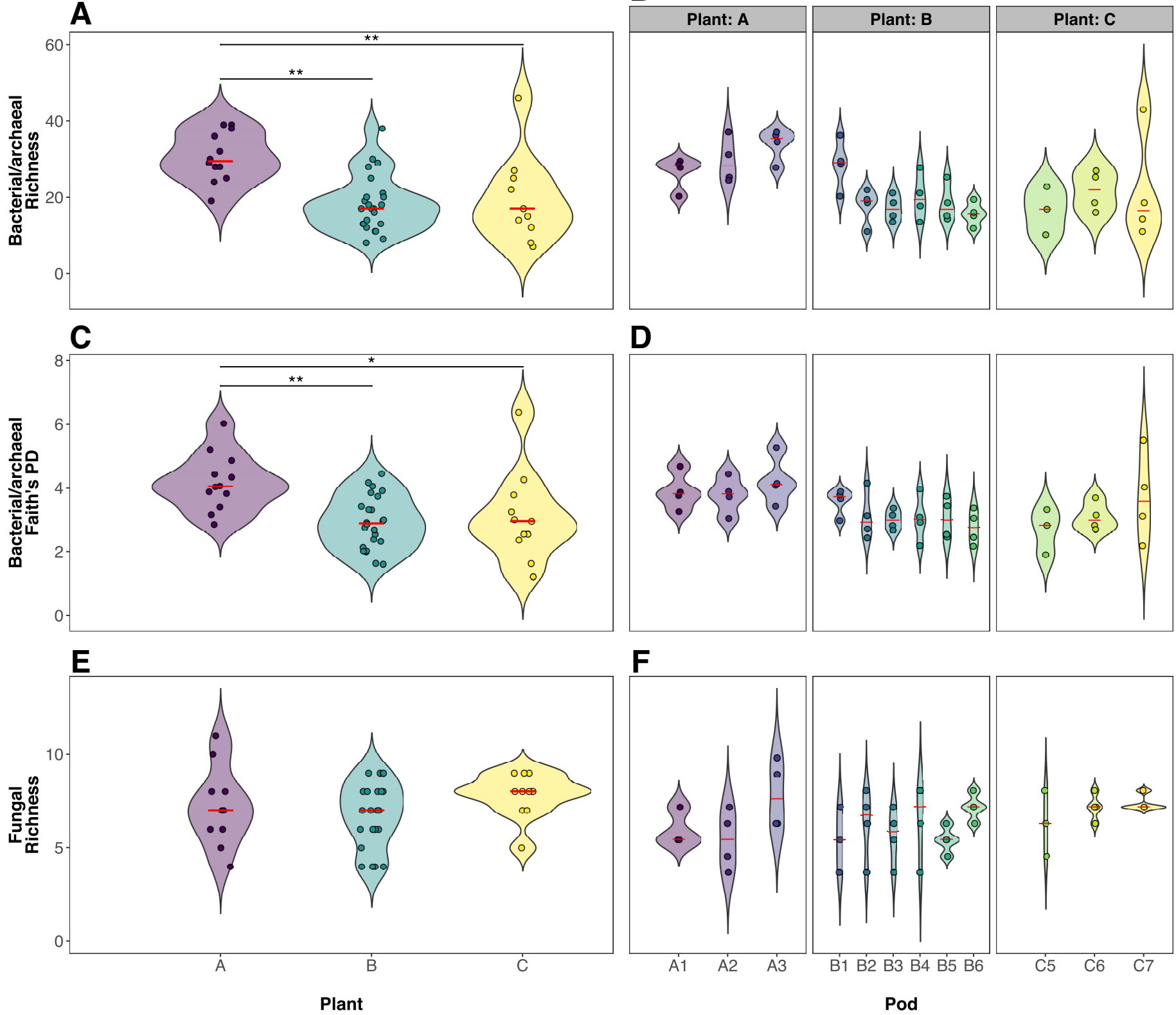

Fig. 2. A, Bacterial or archaeal richness in seeds among plants were different (linear mixed-effects model [LMM] $P$ value $=0.015$ ) B, but not among pods within plant $(P$ value $>0.05)$. C, Bacterial or archaeal phylogenetic diversity in seeds among plants were different $(L M M P$ value $=0.003)$ D, but not among pods within a plant $(P$ value $>0.05)$. E, Fungal richness in seeds was not different among plants (LMM $P$ value $=0.37)$ and $\mathbf{F}$, among pods within a plant $(P$ value $>0.05)$. Here, each point represents the microbiome richness from a microbial DNA extraction from an individual seed. 
is prominent. However, recent work has suggested that the endophytic seed microbiome of green bean varieties of common bean likely colonize predominantly via the internal vascular pathway, and not the floral pathway (Chesneau et al. 2020), which may result in more homogeneity among seed microbiomes of the same plant. Our data support this finding, because seeds from different pods in the same plant (and, therefore, a common vascular pathway across pods) had relatively low microbiome variability, especially compared across plants. It is expected that the vascular pathway of seed microbiome assembly is more likely to colonize the internal seed compartments (e.g., embryo) and, therefore, more likely to be vertically transmitted (Barret et al. 2016). It is as-yet unclear whether plant species that have a stronger relative importance of the floral pathway in seed microbiome assembly may exhibit higher microbiome variability at the pod or fruit level. Such an outcome may indicate that the experimental unit should, instead, be the pod level rather than the plant level for plant species dominated by floral assembly pathways.

There are many challenges in analyzing the microbiome of seeds generally and of a single seed in particular, which may be why cultivation-independent studies of single seeds are few (Abdelfattah et al. 2021). Previous studies showed that seeds have low microbial biomass and diversity (Adam et al. 2018; Chesneau et al. 2020; Rezki et al. 2016), especially relative to other plant compartments or soil. Therefore, many studies pool seeds to analyze the aggregated microbiome of many seeds and to get enough microbial biomass for microbial DNA extraction (Adam et al. 2018; Bergna et al. 2018;
A Bacteria/archaea

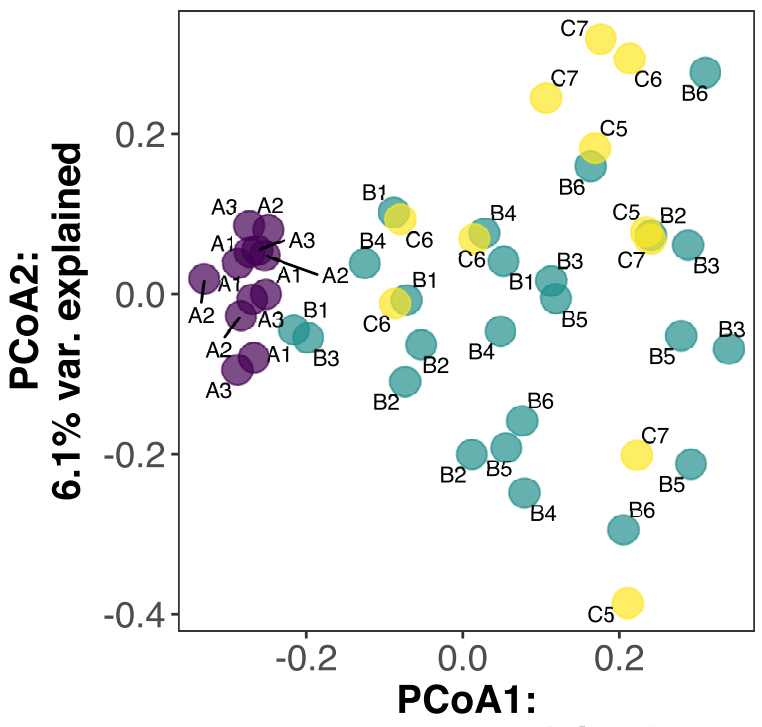

$12.3 \%$ var. explained

C

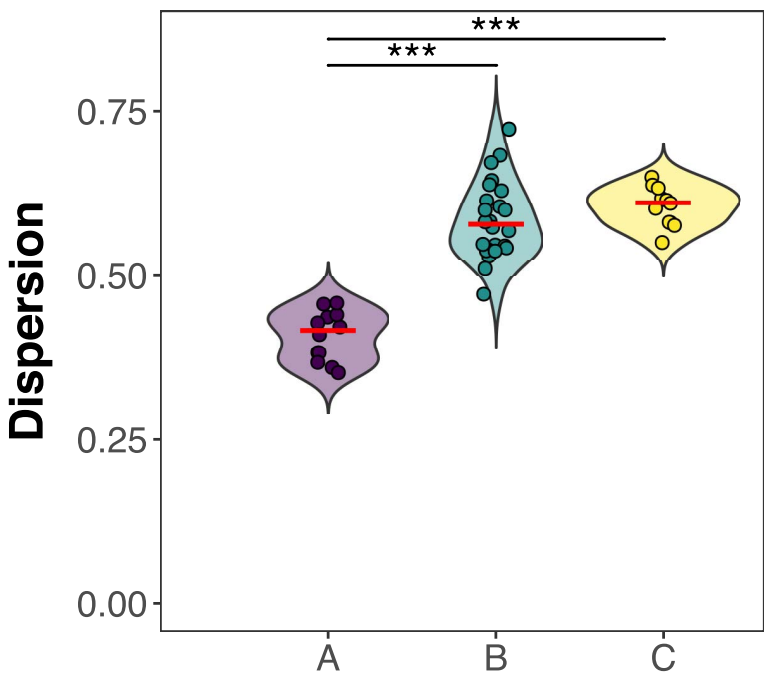

B Fungi

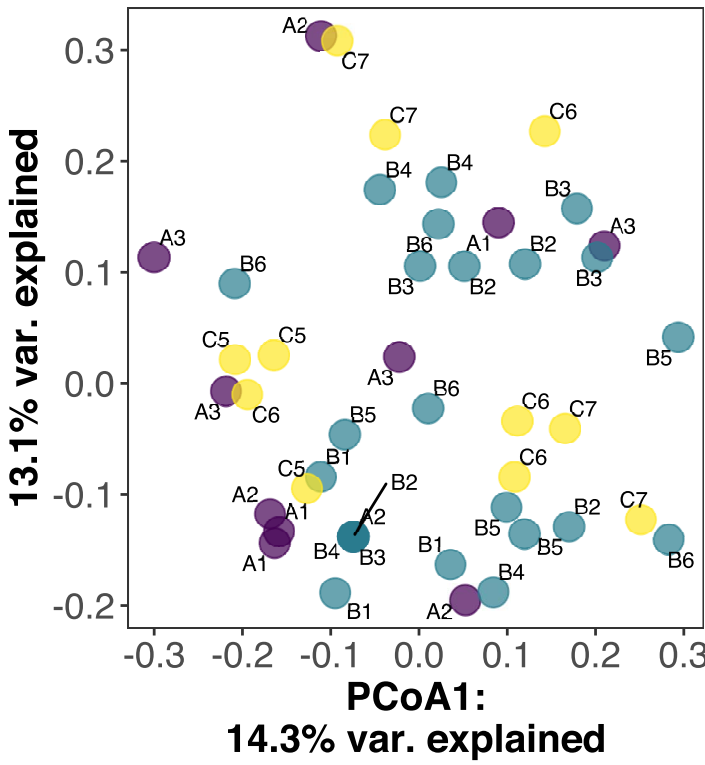

D

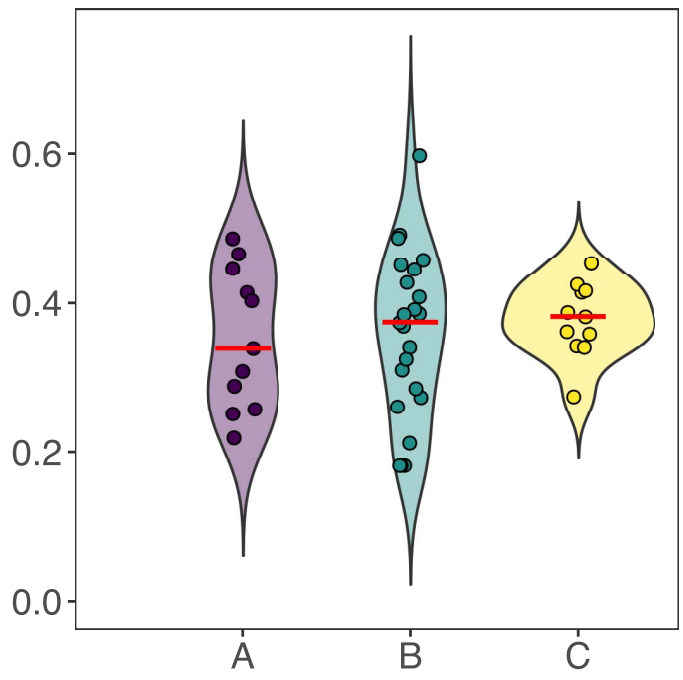

Plant

\section{$14.3 \%$ var. explained}

Plant and Pod

$A$ (Pod A1:A3)

$B$ (Pod B1:B6)

C (Pod C5:C7) 
Johnston-Monje and Raizada 2011; Klaedtke et al. 2016; Latz et al. 2021; Wassermann et al. 2019). Generally, microbiome samples that have low biomass have numerous challenges in sequence-based analysis, as discussed elsewhere (Bender et al. 2018; Eisenhofer et al. 2019). First, unknown contaminants, either from nucleic acid kits or from mishandling of the samples, can have relatively high impact on the observed community composition and, thus, extraction and PCR controls are needed for assessment of contaminants and subtraction of suspected contaminants from the resulting community (Davis et al. 2018). Second, the sparse datasets (e.g., many zero observations for many taxa in many samples) generated from low-biomass samples often require special statistical consideration and data normalization (Anderson et al. 2011; Weiss et al. 2017).
Plant host contamination of the microbiome sequence data are another consideration expected with analysis of the seed, and this challenge also applies to other plant compartments (Fitzpatrick et al. 2018; Song and Xie 2020). For 16S rRNA amplicon sequencing, the contaminant reads typically derive from host mitochondria and chloroplasts but rRNA ITS2 or 18S amplicon analysis may also have reads annotated as Plantae. Therefore, nucleic acid extractions may attempt minimal disturbance of the plant tissue that is the target of microbiome investigation; for example, grinding tissues to include in the extraction will result in higher plant DNA contamination than separating microbial biomass from intact tissue. The cost of this is that any microbes lodged tightly into the host tissue or persisting within host cells may be missed.

\section{A Bacteria/archaea}
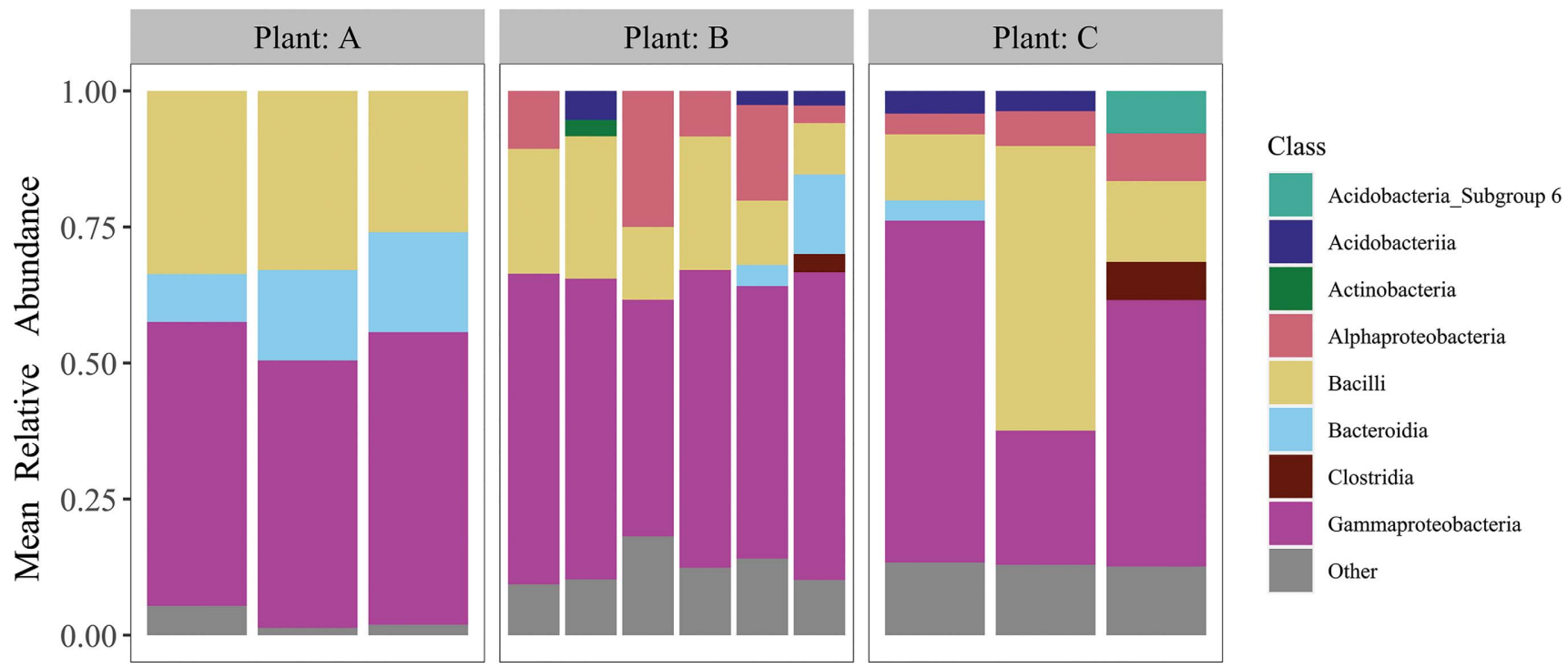

\section{B Fungi}
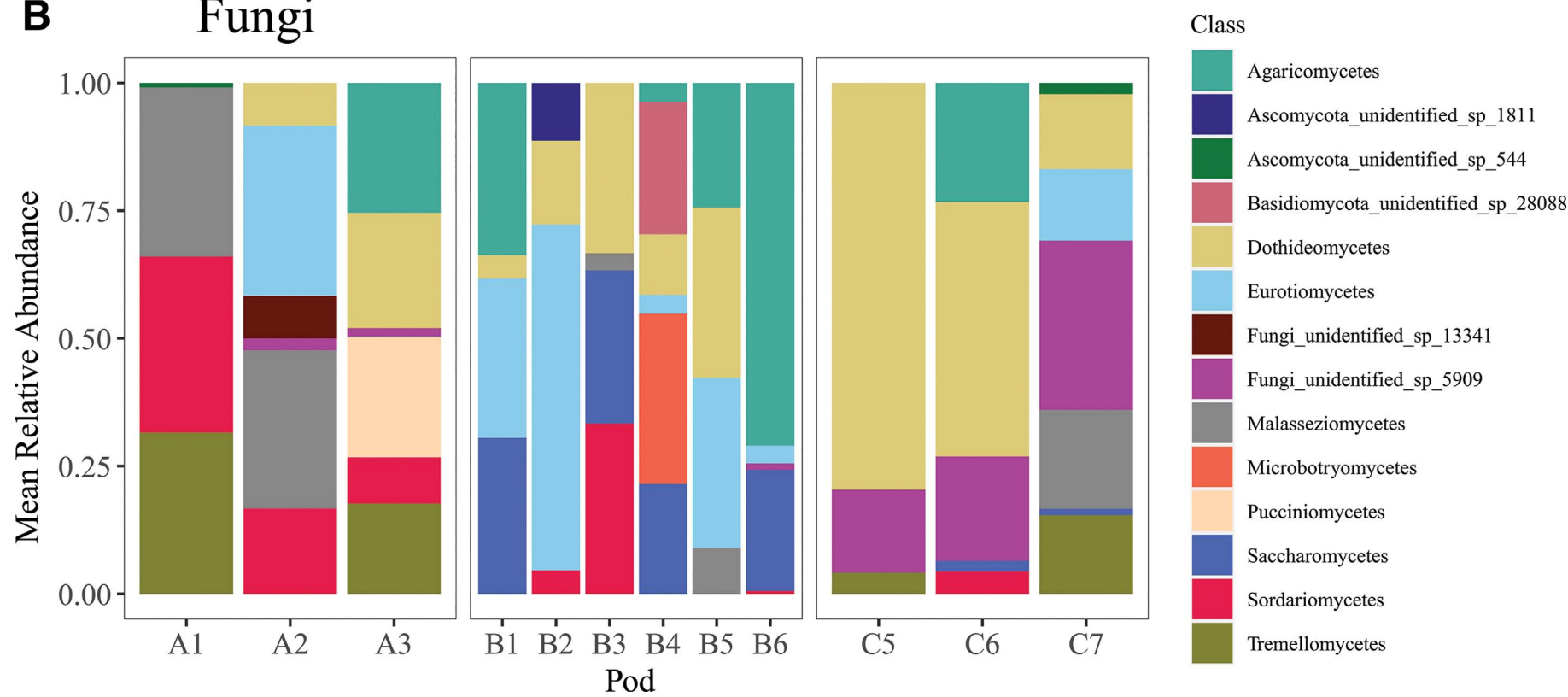

Fig. 4. Bar plots represent mean relative abundances of $\mathbf{A}$, bacterial or archaeal and $\mathbf{B}$, fungal classes in seeds detected across plants. Each bar shows the average composition of individual seeds that were each extracted and analyzed from the same pod. For bacteria or archaea, each pod consisted of four seeds (except for C5; three seeds); and, for fungi, each pod consisted of three seeds (except for A3, B6, C6, and C7: four seeds). The endophyte microbiome was assessed from the DNA extracted from a single seed collected from each pod. Bacterial or archaeal and fungal classes with mean relative abundances of less than $10 \%$ were grouped into the "Other" classification, which includes many lineages (not monophyletic). 
A

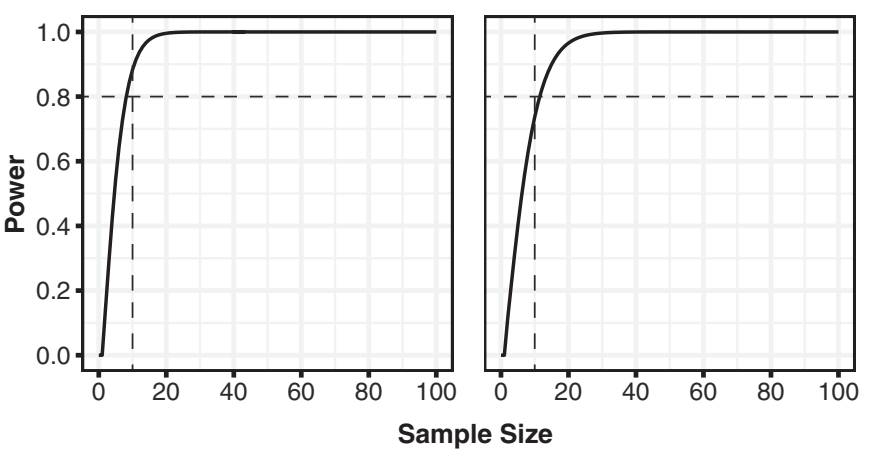

Fig. 5. Analysis of power revealed that an effect of treatment on the 16 S ribosomal RNA bacterial or archaeal A, $\alpha$ diversity (richness) and B, phylogenetic diversity would be detectable in 12 plants at a power of 0.8 . Because the highest seed microbiome variability was at the parent plant level, individual seed microbiome sequence profiles were pooled in silico by plant to perform this power analysis at the individual plant level.

For our study, we wished to understand the microbiome with which a dormant seed begins. This is a key aspect of our approach, because it is known that seeds can exude both antimicrobials and attractants to select for particular microbial members early in microbiome assembly of the germinated seed and emerging seedling (Chesneau et al. 2020; Meldau et al. 2012), and there is an active zone of plant and microbiome activity at the seed-soil interface of a germinating seed (the spermosphere) (Schiltz et al. 2015).Therefore, to target the native endophytic seed microbiome without also allowing the seeds or seedling to select or filter particular members, we used dormant seeds and took care to minimally disrupt their tissues. Notably, many protocols have opted to first germinate seeds and, therefore, include the outcome of early plant selection on the observed seed microbiome (Bergna et al. 2018; López-López et al. 2010; Parsa et al. 2016; Wassermann et al. 2021). Though there are advantages and disadvantages to both germinated and dormant seed microbiome assessment, we reason that focus on the endophyte of the dormant seed is more likely to characterize taxa that have been transmitted from parent to seed.

Taking all of these methodological aspects into consideration, this study presents a protocol and analysis pipeline for endophyte microbiome DNA extraction from a single dormant seed that experiences minimal tissue disruption in the extraction process, includes both positive and negative sequencing controls, and includes bioinformatic steps to identify contamination and remove host signal from the marker gene amplification. Notably, we chose to perform microbiome analysis based on a presence or absence (unweighted) taxon table rather than a table with relativized (weighted) taxon abundances. This was done in consideration of the ecology of the seed endophyte microbiome members to likely be dormant until germination (Cope-Selby et al. 2017) and, therefore, the differences in relativized abundances do not reflect differences in fitness outcomes inside the dormant seed. We acknowledge that relative abundances could reflect differential microbiome member recruitment by the host plant during seed formation; however, this is not the objective of the study and would be best addressed with a different design to determine the multigeneration consistency and transmission rates of any observed enrichments, which would be supported by assessment of the seed microbiome within individual seeds, and across plant generations. Finally, we acknowledge that the relatively prominent size of the edible common bean seed was to our study's advantage, and that some other seeds (e.g., from some dicots) may not be as accessible for sampling via this protocol as individual units because of their small size, structure, and challenges in removing the seed coat.

In conclusion, individual seed microbiome assessment provides improved precision in our understanding of plant microbiome assembly and sets the stage for studies of vertical transmission. We found that seeds produced by an individual bean plant can be considered as a unit (for comparative treatment study designs), and that seeds produced by different plants are expected to have slightly different microbiomes, even if grown under the same, controlled conditions and in the same soil source. Future work may consider whether functional redundancy in plant-beneficial phenotypes across seed microbiome members may provide one mechanism for consistent outcomes in beneficial plant microbiome establishment.

\section{NOTE ADDED IN PROOF}

New information regarding issues with the ITS annotation tool CONSTAX v1 was discovered after the manuscript was accepted. Briefly, the CONSTAX v1 consensus taxonomy assigned fungal genus-level annotations to Phaseolus vulgaris (plant host) ITS2 reads. We have identified the misannotated plant reads with NCBI BLAST and removed them, with updated and annotated workflow in the linked GitHub repository. Changes have been made to this article to reflect this new information.

\section{LITERATURE CITED}

Abdelfattah, A., Wisniewski, M., Schena, L., and Tack, A. J. M. 2021. Experimental evidence of microbial inheritance in plants and transmission routes from seed to phyllosphere and root. Environ. Microbiol. 23:2199-2214.

Adam, E., Bernhart, M., Müller, H., Winkler, J., and Berg, G. 2018. The Cucurbita pepo seed microbiome: Genotype-specific composition and implications for breeding. Plant Soil 422:35-49.

Anderson, M. J., Crist, T. O., Chase, J. M., Vellend, M., Inouye, B. D., Freestone, A. L., Sanders, N. J., Cornell, H. V., Comita, L. S., Davies, K. F., Harrison, S. P., Kraft, N. J. B., Stegen, J. C., and Swenson, N. G. 2011. Navigating the multiple meanings of $\beta$ diversity: A roadmap for the practicing ecologist. Ecol. Lett. 14:19-28.

Barret, M., Briand, M., Bonneau, S., Préveaux, A., Valière, S., Bouchez, O., Hunault, G., Simoneau, P., and Jacques, M.-A. 2015. Emergence shapes the structure of the seed microbiota. Appl. Environ. Microbiol. 81:1257-1266.

Barret, M., Guimbaud, J. F., Darrasse, A., and Jacques, M. A. 2016. Plant microbiota affects seed transmission of phytopathogenic microorganisms. Mol. Plant Pathol. 17:791-795.

Bender, J. M., Li, F., Adisetiyo, H., Lee, D., Zabih, S., Hung, L., Wilkinson, T. A., Pannaraj, P. S., She, R. C., Bard, J. D., Tobin, N. H., and Aldrovandi, G. M. 2018. Quantification of variation and the impact of biomass in targeted 16S rRNA gene sequencing studies. Microbiome 6:155.

Berg, G., and Raaijmakers, J. M. 2018. Saving seed microbiomes. ISME J. 12:1167-1170.

Bergna, A., Cernava, T., Rändler, M., Grosch, R., Zachow, C., and Berg, G. 2018. Tomato seeds preferably transmit plant beneficial endophytes. Phytobiomes J. 2:183-193.

Bintarti, A. F., Kearns, P. J., Sulesky, A., and Shade, A. 2020. Abiotic treatment to common bean plants results in an altered seed microbiome. bioRxiv.

Caporaso, J. G., Bittinger, K., Bushman, F. D., Desantis, T. Z., Andersen, G. L., and Knight, R. 2010a. PyNAST: A flexible tool for aligning sequences to a template alignment. Bioinformatics 26:266-267. Caporaso, J. G., Kuczynski, J., Stombaugh, J., Bittinger, K., Bushman, F. D., Costello, E. K., Fierer, N., Peña, A. G., Goodrich, J. K., Gordon, J. I., Huttley, G. A., Kelley, S. T., Knights, D., Koenig, J. E., Ley, R. E., Lozupone, C. A., McDonald, D., Muegge, B. D., Pirrung, M., Reeder, J., Sevinsky, J. R., Turnbaugh, P. J., Walters, W. A., Widmann, J., Yatsunenko, T., Zaneveld, J., and Knight, R. 2010b. QIIME allows analysis of high-throughput community sequencing data. Nat. Methods 7:335-336.

Caporaso, J. G., Lauber, C. L., Walters, W. A., Berg-Lyons, D., Lozupone, C. A., Turnbaugh, P. J., Fierer, N. and, Knight, M. 2011. Global patterns of $16 \mathrm{~S}$ rRNA diversity at a depth of millions of sequences per sample. Proc. Natl. Acad. Sci. U.S.A. 108:4516-4522. 
Castro-Guerrero, N., Isidra-Arellano, M., Mendoza-Cozatl, D., and Valdés-López, O. 2016. Common bean: A legume model on the rise for unraveling responses and adaptations to iron, zinc, and phosphate deficiencies. Front. Plant Sci. 7:600.

Chartrel, V., Dugat-Bony, E., Sarthou, A. S., Huchette, S., Bonnarme, P., and Irlinger, F. 2021. The microbial community associated with pea seeds (Pisum sativum) of different geographical origins. Plant Soil 462:405-427.

Chesneau, G., Torres-Cortes, G., Briand, M., Darrasse, A., Preveaux, A., Marais, C., Jacques, M.-A., Shade, A., and Barret, M. 2020. Temporal dynamics of bacterial communities during seed development and maturation. FEMS Microbiol. Ecol. 96:fiaa190.

Cole, J. R., Wang, Q., Fish, J. A., Chai, B., McGarrell, D. M., Sun, Y., Brown, C. T., Porras-Alfaro, A., Kuske, C. R., and Tiedje, J. M. 2014. Ribosomal Database Project: Data and tools for high throughput rRNA analysis. Nucleic Acids Res. 42:D633-D642.

Cope-Selby, N., Cookson, A., Squance, M., Donnison, I., Flavell, R., and Farrar, K. 2017. Endophytic bacteria in Miscanthus seed: Implications for germination, vertical inheritance of endophytes, plant evolution and breeding. GCB Bioenergy 9:57-77.

Dai, Y., Li, X.-Y., Wang, Y., Li, C.-X., He, Y., Lin, H.-H., Wang, T., and Ma, X.-R. 2020. The differences and overlaps in the seed-resident microbiome of four Leguminous and three Gramineous forages. Microb. Biotechnol. 13:1461-1476.

Davis, N. M., Proctor, D. M., Holmes, S. P., Relman, D. A., and Callahan, B. J. 2018. Simple statistical identification and removal of contaminant sequences in marker-gene and metagenomics data. Microbiome 6:226.

Edgar, R. C. 2010. Search and clustering orders of magnitude faster than BLAST. Bioinformatics 26:2460-2461.

Edgar, R. C. 2013. UPARSE: Highly accurate OTU sequences from microbial amplicon reads. Nat. Methods 10:996-998.

Edgar, R. C., and Flyvbjerg, H. 2015. Error filtering, pair assembly and error correction for next-generation sequencing reads. Bioinformatics 31:34763482 .

Eisenhofer, R., Minich, J. J., Marotz, C., Cooper, A., Knight, R., and Weyrich, L. S. 2019. Contamination in low microbial biomass microbiome studies: Issues and recommendations. Trends Microbiol. 27:105-117.

Eyre, A. W., Wang, M., Oh, Y., and Dean, R. A. 2019. Identification and characterization of the core rice seed microbiome. Phytobiomes J. 3:148-157.

Fitzpatrick, C. R., Lu-Irving, P., Copeland, J., Guttman, D. S., Wang, P. W., Baltrus, D. A., Dlugosch, K. M., and Johnson, M. T. J. 2018. Chloroplast sequence variation and the efficacy of peptide nucleic acids for blocking host amplification in plant microbiome studies. Microbiome 6:144.

Gdanetz, K., Benucci, G. M. N., Vande Pol, N., and Bonito, G. 2017. CONSTAX: A tool for improved taxonomic resolution of environmental fungal ITS sequences. BMC Bioinf. 18:538.

Hoagland, D. R., and Arnon, D. I. 1950. The water-culture method for growing plants without soil. Circ. Calif. Agric. Exp. Stn. 347:32.

Johnston-Monje, D., and Raizada, M. N. 2011. Conservation and diversity of seed associated endophytes in Zea across boundaries of evolution, ethnography and ecology. PLoS One 6:e20396.

Kelly, J. D., Hosfield, G. L., Varner, G. V., Uebersax, M. A., Long, R. A., and Taylor, J. 1998. Registration of 'Red Hawk'. Dark Red Kidney Bean. Crop Sci. 38:280-281.

Kindt, R. 2020. BiodiversityR: Package for Community Ecology and Suitability Analysis. R package version 2.13-1. https://cran.r-project.org/ web/packages/BiodiversityR/index.html

Klaedtke, S., Jacques, M.-A., Raggi, L., Préveaux, A., Bonneau, S., Negri, V., Chable, V., and Barret, M. 2016. Terroir is a key driver of seedassociated microbial assemblages. Environ. Microbiol. 18:1792-1804.

Latz, M. A. C., Kerrn, M. H., Sørensen, H., Collinge, D. B., Jensen, B., Brown, J. K. M., Madsen, A. M., and Jørgensen, H. J. L. 2021. Succession of the fungal endophytic microbiome of wheat is dependent on tissue-specific interactions between host genotype and environment. Sci. Total Environ. 759:143804.

López-López, A., Rogel, M. A., Ormeño-Orrillo, E., Martínez-Romero, J., and Martínez-Romero, E. 2010. Phaseolus vulgaris seed-borne endophytic community with novel bacterial species such as Rhizobium endophyticum sp. nov. Syst. Appl. Microbiol. 33:322-327.

Martin, M. 2011. Cutadapt removes adapter sequences from high-throughput sequencing reads. EMBnet. J. 17:10-12.

McKnight, D. T., Huerlimann, R., Bower, D. S., Schwarzkopf, L., Alford, R. A., and Zenger, K. R. 2019. microDecon: A highly accurate read-subtraction tool for the post-sequencing removal of contamination in metabarcoding studies. Environ. DNA 1:14-25

McMurdie, P. J., and Holmes, S. 2013. Phyloseq: An R package for reproducible interactive analysis and graphics of microbiome census data. PLoS One 8:e61217.

Meldau, D. G., Long, H. H., and Baldwin, L. T. 2012. A native plant growth promoting bacterium, Bacillus sp. B55, rescues growth performance of an ethylene-insensitive plant genotype in nature. Front. Plant Sci. 3:112.

Mitter, B., Pfaffenbichler, N., Flavell, R., Compant, S., Antonielli, L., Petric, A., Berninger, T., Naveed, M., Sheibani-Tezerji, R., von Maltzahn, G., and Sessitsch, A. 2017. A new approach to modify plant microbiomes and traits by introducing beneficial bacteria at flowering into progeny seeds. Front. Microbiol. 8:11.

Nelson, E. B. 2018. The seed microbiome: Origins, interactions, and impacts. Plant Soil 422:7-34.

Oksanen, J., Blanchet, F. G., Friendly, M., Kindt, R., Legendre, P., McGlinn, D., Minchin, P. R., O’Hara, R. B., Simpson, G. L., Solymos, P., Stevens, M. H. H., Szoecs, E., and Wagner, H. 2019. vegan: Community Ecology Package. R package version 2.5-7. https://CRAN.R-project.org/ package $=$ vegan

Op De Beeck, M., Lievens, B., Busschaert, P., Declerck, S., Vangronsveld, J., and Colpaert, J. V. 2014. Comparison and validation of some ITS primer pairs useful for fungal metabarcoding studies. PLoS One 9:e97629.

Parsa, S., García-Lemos, A. M., Castillo, K., Ortiz, V., López-Lavalle, L. A. B., Braun, J., and Vega, F. E. 2016. Fungal endophytes in germinated seeds of the common bean, Phaseolus vulgaris. Fungal Biol. 120:783-790.

Paulson, J. N., Colin Stine, O., Bravo, H. C., and Pop, M. 2013. Differential abundance analysis for microbial marker-gene surveys. Nat. Methods 10:1200-1202.

Pinheiro, J., Bates, D., DebRoy, S., Sarkar, D., Heisterkamp, S., Van Willigen, B., and Ranke, J. 2021. nlme: Linear and Nonlinear Mixed Effects Models. R package version 3.1-152. https://cran.r-project.org/web/ packages/nlme/index.html

Quast, C., Pruesse, E., Yilmaz, P., Gerken, J., Schweer, T., Yarza, P., Peplies, J., and Glöckner, F. O. 2013. The SILVA ribosomal RNA gene database project: Improved data processing and web-based tools. Nucleic Acids Res. 41:D590-D596.

Raj, G., Shadab, M., Deka, S., Das, M., Baruah, J., Bharali, R., and Talukar, N. C. 2019. Seed interior microbiome of rice genotypes indigenous to three agroecosystems of Indo-Burma biodiversity hotspot. BMC Genomics 20:924.

Rezki, S., Campion, C., Iacomi-Vasilescu, B., Preveaux, A., Toualbia, Y., Bonneau, S., Briand, M., Laurent, E., Hunault, G., Simoneau, P., Jacques, M.-A., and Barret, M. 2016. Differences in stability of seed-associated microbial assemblages in response to invasion by phytopathogenic microorganisms. PeerJ 4:e1923.

Rezki, S., Campion, C., Simoneau, P., Jacques, M.-A., Shade, A., and Barret, M. 2018. Assembly of seed-associated microbial communities within and across successive plant generations. Plant Soil 422:67-79.

Rideout, J. R., He, Y., Navas-Molina, J. A., Walters, W. A., Ursell, L. K., Gibbons, S. M., Chase, J., McDonald, D., Gonzalez, A., Robbins-Pianka, A., Clemente, J. C., Gilbert, J. A., Huse, S. M., Zhou, H.-W., Knight, R., and Caporaso, J. G. 2014. Subsampled open-reference clustering creates consistent, comprehensive OTU definitions and scales to billions of sequences. PeerJ 2:e545.

Rodríguez, C. E., Antonielli, L., Mitter, B., Trognitz, F., and Sessitsch, A. 2020. Heritability and functional importance of the Setaria viridis bacterial seed microbiome. Phytobiomes J. 4:40-52.

Schiltz, S., Gaillard, I., Pawlicki-Jullian, N., Thiombiano, B., Mesnard, F., and Gontier, E. 2015. A review: What is the spermosphere and how can it be studied? J. Appl. Microbiol. 119:1467-1481.

Shade, A., Jacques, M.-A., and Barret, M. 2017. Ecological patterns of seed microbiome diversity, transmission, and assembly. Curr. Opin. Microbiol. $37: 15-22$.

Shade, A., and Stopnisek, N. 2019. Abundance-occupancy distributions to prioritize plant core microbiome membership. Curr. Opin. Microbiol. 49: 50-58.

Song, L., and Xie, K. 2020. Engineering CRISPR/Cas9 to mitigate abundant host contamination for $16 \mathrm{~S}$ rRNA gene-based amplicon sequencing. Microbiome 8:80.

Truyens, S., Weyens, N., Cuypers, A., and Vangronsveld, J. 2015. Bacterial seed endophytes: Genera, vertical transmission and interaction with plants. Environ. Microbiol. Rep. 7:40-50. 
Vujanovic, V., and Germida, J. J. 2017. Seed endosymbiosis: A vital relationship in providing prenatal care to plants. Can. J. Plant Sci. 97:972-981.

Wang, Q., Garrity, G. M., Tiedje, J. M., and Cole, J. R. 2007. Naïve Bayesian classifier for rapid assignment of rRNA sequences into the new bacterial taxonomy. Appl. Environ. Microbiol. 73:5261-5267.

Wassermann, B., Cernava, T., Müller, H., Berg, C., and Berg, G. 2019. Seeds of native alpine plants host unique microbial communities embedded in cross-kingdom networks. Microbiome 7:108.

Wassermann, B., Rybakova, D., Adam, E., Zachow, C., Bernhard, M., Müller, M., Mancinelli, R., and Berg, G. 2021. Studying seed microbiomes. Pages 1-21 in: The Plant Microbiome. L. C. Carvalhais and
P. G. Dennis, eds. Methods in Molecular Biology, vol. 2232. Humana, New York, NY, U.S.A.

Weiss, S., Xu, Z. Z., Peddada, S., Amir, A., Bittinger, K., Gonzalez, A., Lozupone, C., Zaneveld, J. R., Vázquez-Baeza, Y., Birmingham, A., Hyde, E. R., and Knight, R. 2017. Normalization and microbial differential abundance strategies depend upon data characteristics. Microbiome 5:27.

Xing, H.-Q., Ma, J.-C., Xu, B.-L., Zhang, S.-W., Wang, J., Cao, L., and Yang, X.-M. 2018. Mycobiota of maize seeds revealed by rDNA-ITS sequence analysis of samples with varying storage times. MicrobiologyOpen 7:e00609. 